\title{
Compósitos de Polietileno de Baixa Densidade (PEBD) Reforçados com Sílica e Silano
}

\author{
Fernanda F. Gondim, Letícia J. Oliveira, Ronaldo L. Souza \& Rinaldo F. Luz
}

Compósitos de Polietileno de Baixa Densidade (PEBD), reforçados com sílica precipitada em presença de Viniltrimetoxi Silano (VTMS), destinados a composições de dutos de petróleo, foram obtidos e caracterizados. As composições foram processadas por extrusão variando o teor de sílica na matriz de PEBD em 1\%, 3\% e 5\%, com e sem a presença do VTMS. O comportamento reológico das composições foi investigado durante o processamento, mostrando que a presença do silano provocou redução do valor médio do torque e viscosidade e dispersão da carga. Tais resultados foram corroborados pela Microscopia Eletrônica de Varredura com Espectroscopia de Raios X por Dispersão de Energia (MEV-EDS), exibindo, de um modo geral, melhor dispersão da carga na matriz polimérica e melhor processabilidade. Os resultados permitiram também inferir a possibilidade de ganhos de propriedades mecânicas e dinâmicas para tais composições.

Palavras-chaves: polietileno; PEBD; silícia; silano.

Low density polyethylene (LDPE) composites reinforced with silica precipitated in the presence of vinyltrimethoxy silane (VTMS), destined for petroleum duct compositions, were obtained and characterized. The compositions were processed by extrusion by varying the silica content in the LDPE matrix by $1 \%, 3 \%$ and $5 \%$, with and without the presence of VTMS. The rheological behavior of the compositions was investigated during the processing, showing that the presence of the silane caused a reduction in the mean value of force and viscosity and dispersion of the load. These results were corroborated by Scanning Electron Microscopy with Energy Scattering X-Ray Spectroscopy (SEMEDS), showing, in general, better dispersion of the filler in the polymer matrix and better processability. The results also allowed to infer the possibility of improvement of mechanical and dynamic- mechanical properties for such compositions.

Palavras-chaves: polyethylene; LDPE; silica; silane. 


\section{Introdução}

Dentro os diversos artefatos obtidos a partir de matrizes plásticas reforçados, destacam-se os dutos de petróleo, tais como cabos umbilicais e tubos flexíveis. Estes são fundamentais na exploração de petróleo offshore, pois podem transmitir sinais de controle hidráulico, elétrico e óptico, além de auxiliarem no fluxo de fluidos. Estes dispositivos, de um modo geral, são obtidos por complexos processos de extrusão e apresentam multicamadas dispostas de variadas maneiras ${ }^{1-6}$.

Dentre os variados materiais utilizados na fabricação das multicamadas, destacam-se os polímeros, principalmente ,o polietileno de baixa densidade (PELD), com função importante na vedação, redução de peso, prolongamento de vida útil dos dutos - para citar algumas. Entretanto, este material pode apresentar problemas estruturais, tais como trincas, desgaste por abrasão e degradação, ocasionados devido ao manuseio durante o transporte e instalação destes dutos, bem como por correntes marítimas, baixa resistência à temperatura e pressão, além da ausência de isolamento térmico adequado. É sabido que o uso de cargas de reforço dispersas na matriz polimérica contribui para a melhora de propriedades físicas, mecânicas, químicas e térmicas de compósitos poliméricos. No caso dos dutos de petróleo, as propriedades de resistência à fadiga (tensão e flexão), estabilidade dimensional e a resistência à abrasão tendem a aumentar com o uso de carga de reforço, se uma boa dispersão da carga na matriz for mantida.

A literatura mostra que apesar de a sílica contribuir para o ganho das propriedades já citadas, possui grande tendência a se aglomerar, em função de sua polaridade e difícil processabilidade $^{1-3}$. Sob tal condição, perdas significativas de propriedades podem se observadas no compósito. Assim, o uso de agentes de acoplamento, tais como os silanos, tem sido largamente investigado pois contribuem para melhor interação carga-polímero o que, consequentemente, irá favorecer não somente o processamento como o melhor desempenho geral do composto. Este trabalho busca avaliar a contribuição da presença do silano Viniltrimetoxi Silano durante a processabilidade das composições contendo PEBD reforçados com sílica.

\section{Metodologia Experimental}

Foi utilizado PEBD (especificado pela Norma API 2012), cedido pela empresa Oceaneering Basil; sílica Tixosil 333 da Rhodia-Solvay e Vinil Trimetoxi Silano 98\% da Sigma-Aldrich. As composições, conforme descritas na Tabela 1, foram extrusadas em miniextrusora de bancada Thermo Scientific Haake MiniLab II Micro Compounder, sob temperatura média de $150^{\circ} \mathrm{C}$, velocidade de $60 \mathrm{rpm}$ e tempo de residência de 10 minutos. As microscopias foram realizadas em um microscópio Philips-XL30, previamente metalizadas num metalizado catódico (Figura 1). Os corpos de prova para o MEV-EDS foram obtidos por fusão das amostras extrusadas em molde de dimensões de $15 \mathrm{~cm} \mathrm{x}$ $15 \mathrm{~cm} \times 2 \mathrm{~cm}$ (Figura 2), prensados pela prensa hidráulica da Carver Laboratory, modelo B\&C NS 34000-623, sob temperatura de $150^{\circ} \mathrm{C}$ durante 10 minutos, sendo resfriada a $20^{\circ} \mathrm{C}$ e aclimatadas sob mesma temperatura.

\section{Resultados e Discussões}

O perfil da viscosidade é importante para a determinação da melhor condição de processamento, em particular sob altas taxas de cisalhamento, como é o caso dos compostos submetidos aos processos de extrusão. Uma comparação entre as Figuras 3 e 4 mostra que a viscosidade das composições da Série A, contendo o Viniltrimetoxi Silano (VTMS) são menores, do que as composições da Série $\mathrm{B}$, que não contém silano ${ }^{1-6}$.

A comparação entre os resultados de viscosidade do composto $5 \mathrm{~A}$ e $5 \mathrm{~B}$ mostram uma redução de cerca de $10 \%$ na viscosidade desse último e, que de um modo geral, todos os compostos da Série B apresentaram resultados semelhantes. Tal evento pode ser justificado pela forte interação entre os grupos silanóis do silano com as hidroxilas da sílica que contribuem para a quebra das interações carga-carga e promoção da interface polímero-carga, que cresce em função do tempo. Sob tal condição, os agregados de sílica, naturalmente formados devido à forte interação entre si, são reduzidas, resultando em menor volume da carga aglomerada e consequentemente uma menor viscosidade do composto final. Assim, pode-se assumir que grande parte da sílica está dispersa na matriz com o silano VTMS. 


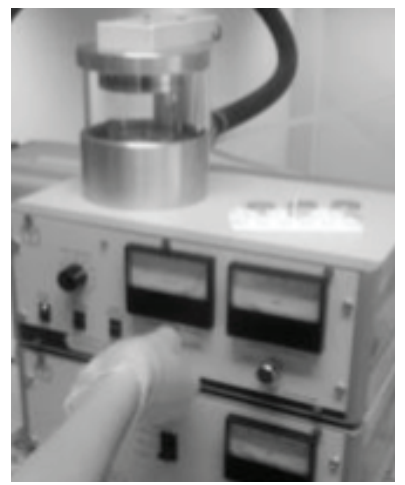

(a)

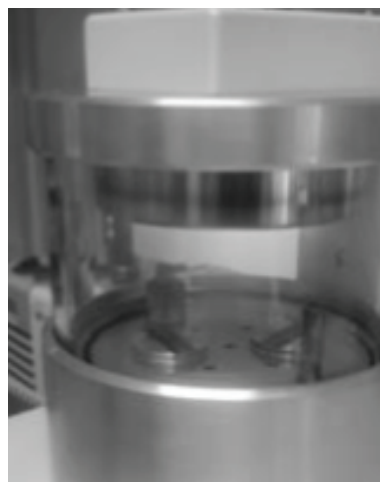

(b)

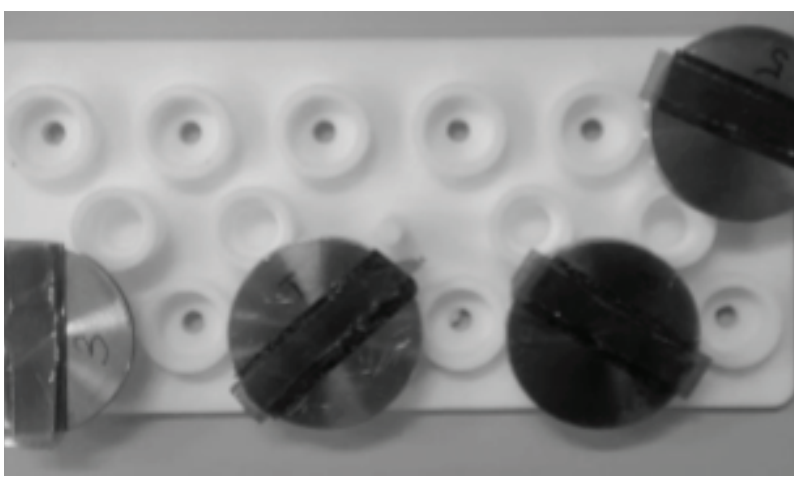

(c)

Figura 1. Pulverizador Catódico. (a) Antes do procedimento, (b) Em operação e (c) Amostras no suporte após serem metalizadas no pulverizador catódico.

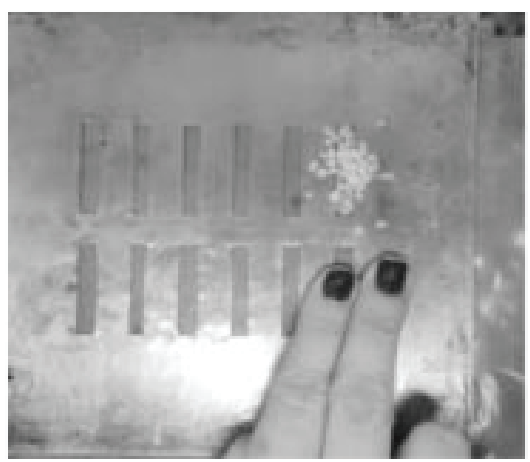

(a)

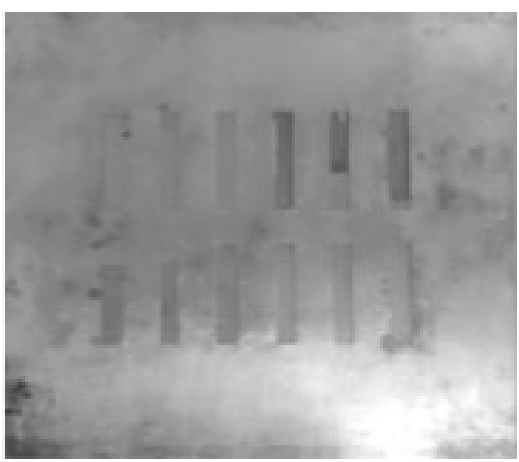

(b)

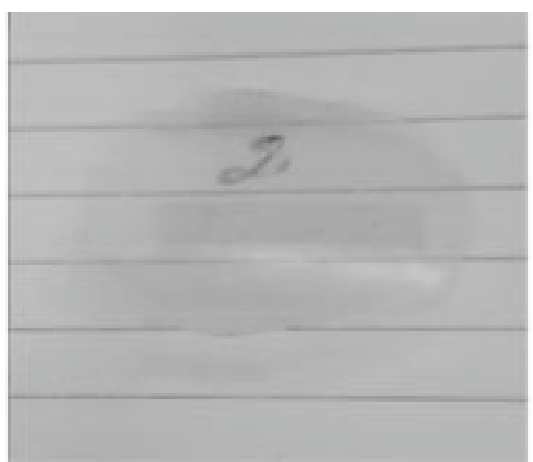

(c)

Figura 2. Preparo dos corpos de prova para o Microscópio Eletrônico de Varredura (MEV). (a) Material picotado; (b) Material no molde após a prensa e (c) Corpo de prova.

Tabela 1. Composição dos compostos com sílica e silano (1A, 3A, 5A) e sem silano (1B, 3B, 5B).

\begin{tabular}{|c|c|c|c|c|c|c|c|c|c|c|c|c|}
\hline \multirow{2}{*}{ Componente } & \multicolumn{2}{|c|}{$1 \mathrm{~A}$} & \multicolumn{2}{c|}{$1 \mathrm{~B}$} & \multicolumn{2}{c|}{$3 \mathrm{~A}$} & \multicolumn{2}{c|}{$3 \mathrm{~B}$} & \multicolumn{3}{c|}{$5 \mathrm{~A}$} & \multicolumn{2}{c|}{$5 \mathrm{~B}$} \\
\cline { 2 - 16 } & $\%$ & $\mathrm{~g}$ & $\%$ & $\mathrm{~g}$ & $\%$ & $\mathrm{~g}$ & $\%$ & $\mathrm{~g}$ & $\%$ & $\mathrm{~g}$ & $\%$ & $\mathrm{~g}$ \\
\hline PEBD & $94 \%$ & 4,70 & $94 \%$ & 4,95 & $92 \%$ & 4,60 & $92 \%$ & 4,85 & $90 \%$ & 4,5 & $90 \%$ & 4,75 \\
\hline Sílica & $1 \%$ & 0,05 & $1 \%$ & 0,05 & $3 \%$ & 0,15 & $3 \%$ & 0,15 & $5 \%$ & 0,25 & $5 \%$ & 0,25 \\
\hline Silano & $5 \%$ & 0,25 & - & - & $5 \%$ & 0,25 & - & - & $5 \%$ & 0,25 & - & - \\
\hline
\end{tabular}


As Figura 5 e 6 mostram as curvas de torque (N.m) em função do tempo (min) para os compostos da Série A (com adição de silano) e Série B (sem adição de silano).

Ambas as Figuras 5 e 6 mostraram que a presença do Silano VTMS provoca uma redução do torque em cerca de $25 \%$ (entre os valores encontrados para os composto $5 \mathrm{~A} \mathrm{e}$ o 5B) e sua estabilização em valores menores. Isso ocorre por motivos semelhantes aos citados para a redução da viscosidade, isto é, sob ação mecânica, as redes e aglomerados maiores são rompidos gerando partículas facilmente distribuídas na matriz de PELD que contém o silano. Evento associado ao decréscimo da diferença de energia superficial entre o polímero e a carga, resultando em menor interação carga-carga e maior interação polímero-carga.

A microscopia eletrônica de varredura (MEV-EDS), apresentada na Figura 7, foi utilizada para a obtenção de informações sobre o tipo de morfologia decorrente da magnitude da dispersão resultante do processamento e da intensidade das interações PELD-sílica.

As micrografias da Figura 7 evidenciam que uma contribuição positiva do silano nas amostras contendo $3 \%$ e $5 \%$ de sílica. A Figura 7A mostra certa heterogeneidade e presença de aglomerados maiores, resultado da forte interação que mantém os aglomerados de sílica, os quais não foram desfeitos pelo processamento. Enquanto que as Figuras 7B e 7C mostram que a presença do silano contribuiu para reduzir a influência dessas interações, uma vez que grande parte da sílica mostra-se dispersa em toda a superfície investigada. Este comportamento pode ser atribuído às razões já citadas, isto é, ao aumento da interação carga-matriz causado pela presença do VTMS. Essa breve investigação está de acordo com o previsto na literatura, a qual, também associa melhor dispersão e fixação da carga à matriz polimérica. Mas, também, evidenciam a necessidade de um estudo morfológico mais aprofundado das amostras obtidas com o ganho de propriedades Mecânicas e dinâmicas.

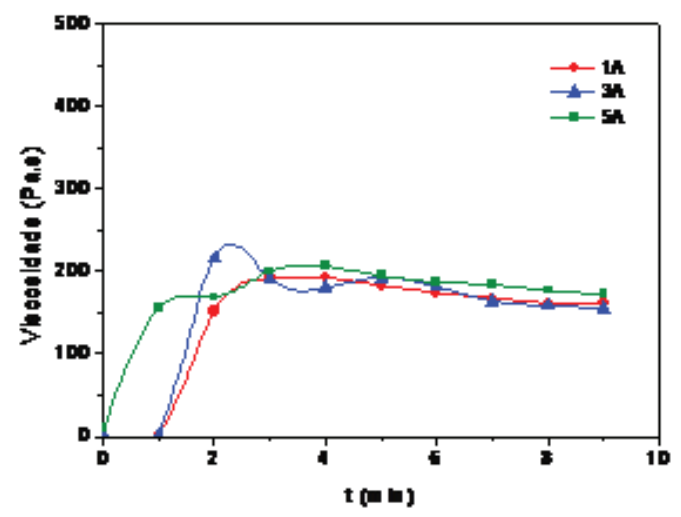

Figura 3. Resultado de Viscosidade (Pa.s) em função do tempo (min) para as composições $1 \mathrm{~A}, 3 \mathrm{~A}, 5 \mathrm{~A}$.

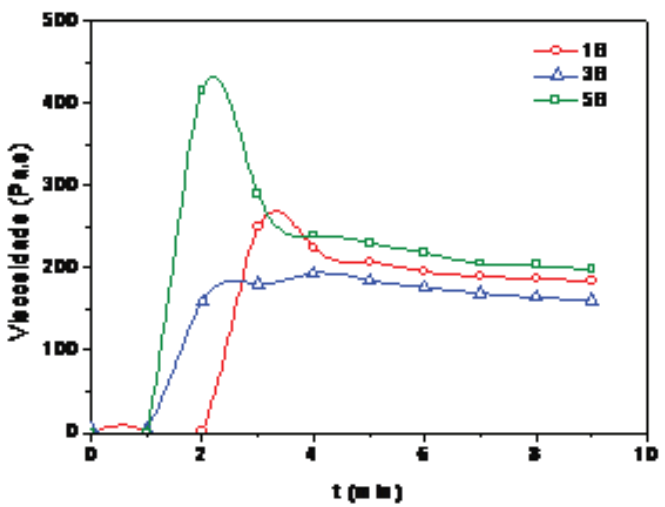

Figura 4. Resultado de Viscosidade (Pa.s) em função do tempo (min) para as composições $1 \mathrm{~B}, 3 \mathrm{~B}, 5 \mathrm{~B}$.

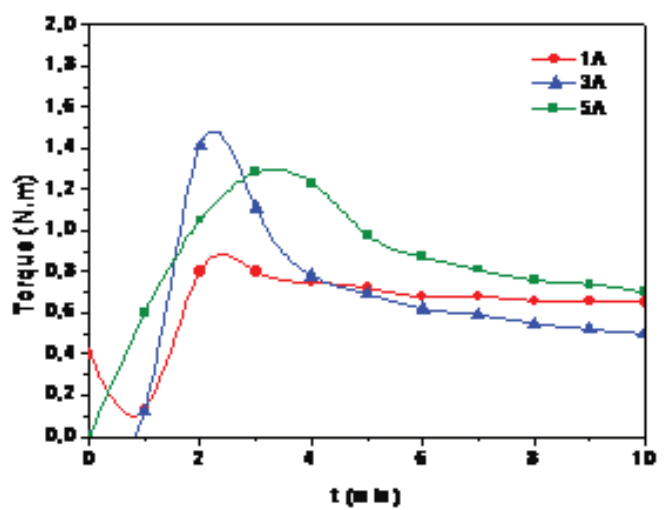

Figura 5. Resultado de Torque (N.m) em função do tempo (min) para as composições 1A, 3A, 5A. 


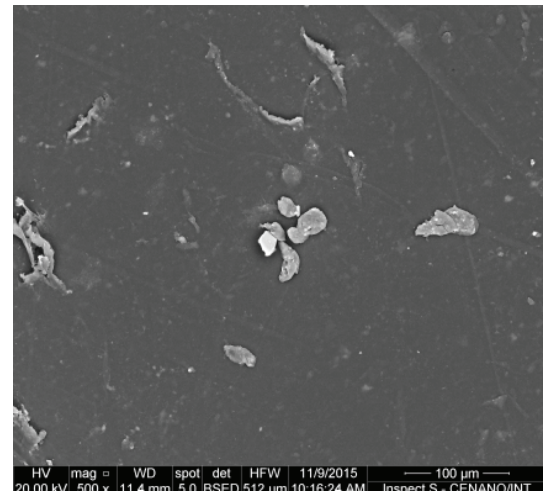

(a)

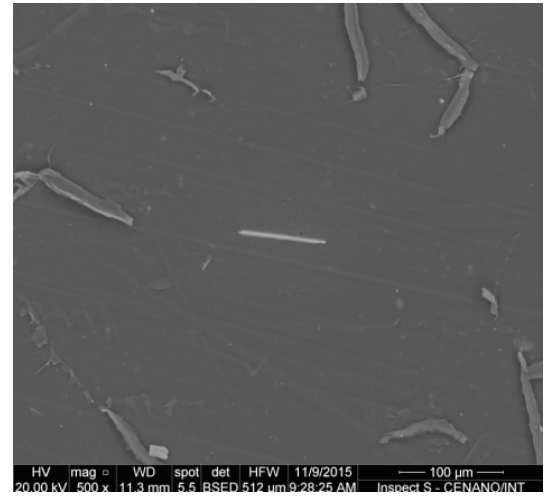

(b)

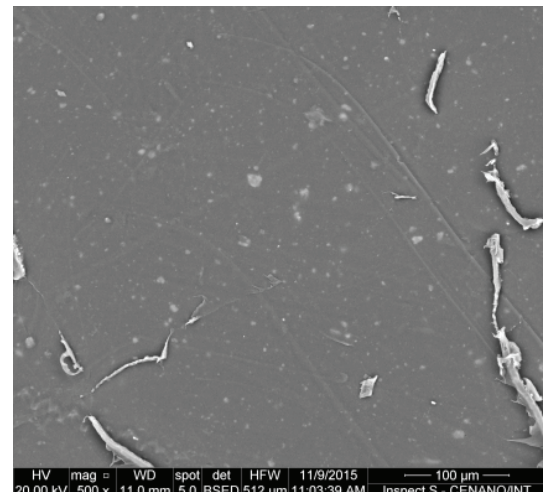

(c)

Figura 7. Micrografias obtidas por MEV para as amostras contendo, respectivamente, 3\% de sílica e sem VTMS (a); 3\% de sílica com VTMS (b) e 5\% de sílica com VTMS (c), com ampliação de 500 x.

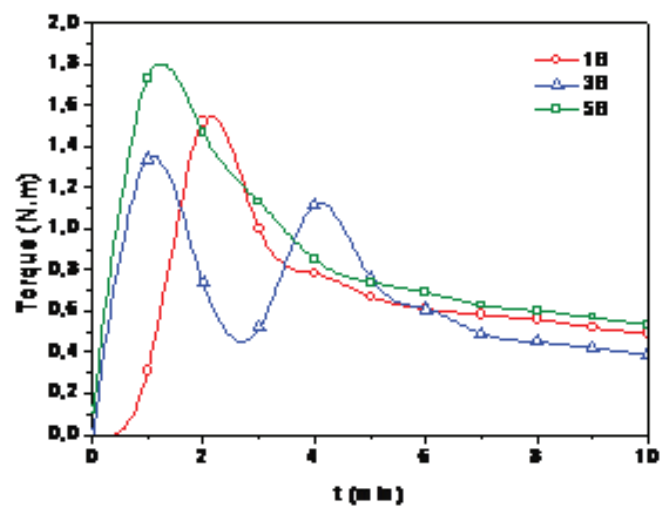

Figura 6. Resultado de Torque (N.m) em função do tempo (min) para as composições 1B, 3B, 5B.

\section{Conclusões}

O uso de sílica com agente de reforço em matrizes poliméricas, apesar de largamente utilizado, necessita de agentes de acoplamento, tais como os silanos. A presença do Viniltrimetoxi Silano (VTMS) na matriz do Polietileno de Baixa Densidade (PEBD) levou à estabilização do torque em valores mais baixos e com menor viscosidade, pois sob ação mecânica, as redes e aglomerados maiores da sílica foram rompidos e facilmente distribuídos na matriz, resultando em um processamento mais efetivo. A Microscopia Eletrônica de Varredura (MEV) corroborou tal observação ao revelar que as superfícies dos compósitos, mesmo com maior teor de sílica, ainda de mostravam homogêneas e com boa dispersam da sílica na matriz de PEBD.

\section{Referências Bibliográficas}

1. Panaitescu, D. M.; Frone, A.n.; Spataru, I.c., Effect of Nanosilica on the morphology of polyethylene investigated by AFM. Comp. Sci. and Tech., v. 74, p. 131-138, 2013.

2. Shieh, Y.; LIU, C., Silane grafting reactions of LDPE, HDPE and LLDPE. J. of Appl. Polym. Sci., v. 74, i.14,p. 3404-3411,1999.

3. Wypych,G., Fillers in Commercial polymers in Handbook of filler, 4th e., p.665-761, NY, Chem. Tec. Publishing, 2016. 


\section{Artigo Geral 7}

4. Katz,H.s.; Mileski, J.V., Handbook of fillers plastics, Springer US, 1988.

5. Fogg,D., Umbilical. Technip France. US 9,343,199 B2,2016.

6. Bectarte, Fabrice; Secher, Philippe; Felix-Henry, Antoine. Qualification testing of flexible pipes for $3000 \mathrm{~m}$ water depth. Offshore Technology Conference. Houston, Texas, 2011.

\section{Fernanda F. Gondim', Letícia J. Oliveira', Ronaldo L. Souza ${ }^{2}$ \& Rinaldo F. Luz ${ }^{1,2 *}$}

${ }^{1}$ Faculdade Senai Cetiqt - Rio de Janeiro, Departamento de Engenharia Química.

${ }^{2}$ Universidade Federal do Rio de Janeiro, Centro de Tecnologia, Escola de Química

*E-mail: rluz@cetiqt.senai.br 